\title{
Aggregated Distance Metric Learning (ADM) for Image Classification in Presence of Limited Training Data
}

\author{
Gaoyu Xiao and Anant Madabhushi* \\ Department of Biomedical Engineering, Rutgers University, USA \\ $\{$ gyxiao, anantm\}@rci.rutgers.edu
}

\begin{abstract}
The focus of image classification through supervised distance metric learning is to find an appropriate measure of similarity between images. Although this approach is effective in the presence of large amounts of training data, classification accuracy will deteriorate when the number of training samples is small, which, unfortunately, is often the situation in several medical applications. We present a novel image classification method called aggregated distance metric (ADM) learning for situations where the training image data are limited. Our approach is novel in that it combines the merits of boosted distance metric learning (BDM, a recently published learning scheme) and bagging theory. This approach involves selecting several sub-sets of the original training data to form a number of new training sets and then performing $\mathrm{BDM}$ on each of these training sub-sets. The distance metrics learned from each of the training sets are then combined for image classification. We present a theoretical proof of the superiority of classification by ADM over BDM. Using both clinical (X-ray) and non-clinical (toy car) images in our experiments (with altogether 10 sets of different parameters) and image classification accuracy as the measure, our method is shown to be more accurate than BDM and the traditional bagging strategy.
\end{abstract}

\section{Introduction}

Image classification is important in many medical applications, for instance, to distinguish images representing different pathologies in the context of contentbased image retrieval (CBIR). Another example is to identify common anatomical landmarks for the purpose of image data fusion and image registration. In general, medical image classification can be explored in two fronts: (1) extracting a representative set of features and (2) finding an appropriate similarity measure between images. The latter, named distance metric learning, is not as extensively explored as the former, it therefore has great potential to further improve the image classification accuracy [1]. Most distance metric learning (DML) methods can be classified as either unsupervised or supervised. The supervised

\footnotetext{
* Thanks to funding agencies: National Cancer Institute (R01CA136535-01, R01CA140772 01, R03CA143991-01), and The Cancer Institute of New Jersey.
}

G. Fichtinger, A. Martel, and T. Peters (Eds.): MICCAI 2011, Part III, LNCS 6893, pp. 33-40, 2011. (C) Springer-Verlag Berlin Heidelberg 2011 
approaches, which require training images with user-defined labels or pair-wise constraints, are more frequently found in medical applications [2].

Boosting framework [3], which uses a set of weak learners to create a strong learner, has been adopted in DML. Recently, a boosted distance metric learning method (BDM) using pair-wise constraints was proposed [1]. This method, when combined with the nearest neighbor search, has been proven to be efficient and accurate in classifying medical images of multiple classes when size of the training data is large enough [1].

However, well annotated training data are almost always difficult to obtain in medical imaging problems. In particular, in supervised multi-class image classification, there may be only limited number of training images for each image class (e.g. a brain atlas can contain a large number of anatomical landmarks derived from a much smaller number of subjects), hence the sample size for each class may not be statistically reasonable [4] 5]. One way to improve the performance of image classification in the presence of limited training images may be via boostrap aggregating (bagging) 6, wherein bootstrapped training sets are constructed and classification is then performed on each set. The final classification is achieved through a plurality vote. However, when the performance of each individual classifier is constrained by the size of the training data, further improvement will be desirable.

In this work, we present a novel method called aggregated distance metric learning $(\mathrm{ADM})$ to classify a test image, specifically in the context of (a) large number of classes, and (b) limited training images for each class. Inspired by the idea of BDM [1] and bagging [6], instead of using the whole training image set $\Omega$ of $k$ classes $C_{i},(i \in\{1, \cdots, k\})$ to derive a single distance metric $d$, our method first selects $M$ sub-sets $\Omega_{m}(m \in\{1, \cdots, M\})$ from $\Omega$, then performs BDM on each $\Omega_{m}$ to obtain a unique distance metric $d_{m}$. In order to determine which class $C^{\prime}$ a test image $t$ belongs to, the distance between $t$ and each $C_{i}$ is computed using every distance metric $d_{m}\left(t, C_{i}\right)$. Next, all $d_{m}$ are aggregated $\sum_{m=1}^{M} d_{m}\left(x, C_{i}\right)$ and the class $C^{\prime}$ with the smallest aggregated distance is identified as the class label. It can be seen that ADM is a meta-algorithm based on BDM. Moreover, it differs from bagging in that bagging conducts a plurality vote on all classification results of each predictor, while ADM computes the aggregated distances from all $d_{m}$ to get the classification result.

To our knowledge, there is no previous work trying to combine the merits of DML and bagging in the context of image classification. In this work, we present a rigorous theoretical analysis to show why ADM yields better classification compared to BDM. In addition, our method is more accurate than the traditional bagging approach, as the continuous aggregated distance value is more robust against the errors caused by small training set sizes. We demonstrate the superiority of ADM over other state of the art methods in experiments involving both clinical (X-ray) and non-clinical (toy car) image data. We also demonstrate the potential applicability of ADM in the context of CBIR applications. 


\section{Previous Related Work}

\subsection{Boosted Distance Metric Learning}

Yang et al proposed BDM for image retrieval and classification 11, where the distance function between data points $x_{1}$ and $x_{2}$ was defined as:

$$
d\left(x_{1}, x_{2}\right)=\sum_{i=1}^{T} \alpha_{i}\left(f_{i}\left(x_{1}\right)-f_{i}\left(x_{2}\right)\right)^{2},
$$

where $f_{i}(x)$ is a binary classification function, and $\alpha_{i}$ are the combination weights, and $T$ is the number of iterations. For the specific task of image classification, $x_{1}$ and $x_{2}$ may present images or extracted features. With the pair-wise constraints, the appropriate classification function $f_{i}(x)$ and the combination weights $\alpha_{i}$ can be learned by using the bound optimization theory 1 .

During image classification, all training images are used to learn distance metric $d$, which is then used to compute the distance between a test image $t$ and each training image. Based on these distance values, the class that is closest to $t$ is considered as the class that the test image should belong to.

\subsection{Bootstrap Aggregating (Bagging)}

In [6] Breiman showed that classification accuracy can be improved by bootstrap sampling of the original data set to generate multiple versions of a classifier, and then using the results from the individual classifiers to obtain a consensus prediction. A theoretical proof was provided in [6] to explain why the bagging classifier, on average, performs better compared to the individual classifiers.

\section{Classification by Aggregated Distance Metric Learning (ADM)}

\subsection{Theoretical Intuition}

Inspired by the work of BDM [1] and bagging [6], ADM integrates the best of both approaches. It first constructs a number of training image sub-sets, then obtains the aggregated distance from these sub-sets for image classification.

\subsection{Constructing Training Image Sub-sets}

We construct $M$ sub-sets of training images from the original training data set $\Omega$. Suppose there are altogether $k$ different classes of training images $C_{1}, \cdots, C_{k}$, and each class $C_{i},(i \in\{1, \cdots, k\})$ consists of $n$ training images $C_{i}=\left\{\left(x_{i j}, y_{i}\right)\right\}$, $(i \in\{1, \cdots, k\} ; j \in\{1, \cdots, n\})$, where $x_{i j}$ is a training image and $y_{i}$ is the corresponding class label. Since in this work we are interested in the scenario where the number of training images for each class, $n$, is small, we assume $k>n$. At each iteration, we randomly choose $n$ classes of training images to form a training image sub-set $\Omega_{m}$ so that we have a total of $M=\left(\begin{array}{l}k \\ n\end{array}\right)$ training image sub-sets $\Omega_{m},(m \in\{1, \cdots, M\})$, where $\Omega_{m}$ consists of all the training images in the $n$ classes that have been chosen. 


\subsection{ADM for Image Classification}

$\mathrm{BDM}$ is performed on each sub-set $\Omega_{m}$ to obtain corresponding distance metric $d_{m}\left(x_{1}, x_{2}\right)$ between two images $x_{1}$ and $x_{2},\left(m \in\left\{1, \cdots,\left(\begin{array}{l}k \\ n\end{array}\right)\right\}\right)$. Given a test image $t$, the aggregated distance between $t$ and a class $C_{i}=\left\{\left(x_{i j}, y_{i}\right)\right\},(i \in\{1, \cdots, k\}$; $j \in\{1, \cdots, n\})$ using $d_{m}$ is defined as:

$$
d_{m}\left(t, C_{i}\right)=\frac{1}{n} \sum_{j=1}^{n} d_{m}\left(t, x_{i j}\right) .
$$

Finally, by summing up all $d_{m},(m \in\{1, \cdots, M\}), M=\left(\begin{array}{l}k \\ n\end{array}\right)$, we get the aggregated distance between image $t$ and class $C_{i}$ as

$$
D\left(t, C_{i}\right)=\sum_{m=1}^{M} d_{m}\left(t, C_{i}\right),
$$

so that the class $C^{\prime}$ with the smallest aggregated distance is chosen as the class which $t$ should belong to. Mathematically, this is formulated as:

$$
C^{\prime}=\arg \min _{i}\left[D\left(t, C_{i}\right)\right] .
$$

Next, we will prove that classification by ADM is better than BDM.

\subsection{Proof of Superiority of Classification by ADM over BDM}

We give a mathematical proof to show that classification by ADM is more accurate compared to BDM.

Preliminaries. We denote the probability that a test image $t$ is classified into class $C$ using the $m$-th distance learning process as $\psi_{m}(t, C), m \in\{1, \cdots, M\}$ $\left(M=\left(\begin{array}{l}k \\ n\end{array}\right)\right.$ if the training image sub-set are constructed according to Section 3.2). We also denote the probability that $t$ is classified into class $C$ using all the distance learning processes (ensemble classifier) as $\Psi(t, C)$.

Theorem. Given that the correct classification of $t$ is class $C_{r}$, then $\Psi\left(t, C_{r}\right) \geq$ $\psi_{m}\left(t, C_{r}\right)$.

Proof. Given that a wrong classification of $t$ is class $C_{w}$, then statistically, it is reasonable to assume

$$
E\left(d_{m}\left(t, C_{w}\right)\right)>E\left(d_{m}\left(t, C_{r}\right)\right),
$$

where $E()$ is the expectation operation. We introduce an auxiliary variable

$$
g_{m}^{t}=d_{m}\left(t, C_{w}\right)-d_{m}\left(t, C_{r}\right) .
$$

Let $E\left(g_{m}^{t}\right)=\mu$ and $\operatorname{var}\left(g_{m}^{t}\right)=\sigma^{2}$. According to Eq. 囵 we have $\mu>0$.

The probability that $t$ is closer to $C_{r}$ when measured by the aggregated distance is

$$
P\left(D\left(t, C_{w}\right)>D\left(t, C_{r}\right)\right)=P\left(\sum_{m=1}^{M} g_{m}^{t}>0\right) .
$$


According to central limit theorem, the distribution of $\sum_{m=1}^{M} g_{m}^{t}$ approaches a normal distribution $N\left(M \mu, \sigma^{2}\right)$ as $M$ becomes larger (since $E\left(g_{m}^{t}\right)=\mu$ and $\left.\operatorname{var}\left(g_{m}^{t}\right)=\sigma^{2}\right)$, so that

$$
E\left(\sum_{m=1}^{M} g_{m}^{t}\right)=M \mu ; E\left(g_{m}^{t}\right)=\mu .
$$

Since given two distributions with the same variance, the one with a larger expectation has a larger probability of being positive, we have

$$
P\left(\sum_{m=1}^{M} g_{m}^{t}>0\right) \geq P\left(g_{m}^{t}>0\right) .
$$

Expanding Eq. 9 using Eq. 6, we have

$$
\begin{aligned}
P\left(\sum_{m=1}^{M} d_{m}\left(t, C_{i}\right)\right. & \left.>\sum_{m=1}^{M} d_{m}\left(t, C_{r}\right)\right) \geq \\
P\left(d_{m}\left(t, C_{i}\right)\right. & \left.>d_{m}\left(t, C_{r}\right)\right), \text { where } i \in\{1, \cdots, r-1, r+1, \cdots, k\}
\end{aligned}
$$

Since probability values are non-negative, from Eq. 10 we have

$$
\begin{gathered}
\prod_{i \neq r, i=1}^{k}\left(P\left(\sum_{m=1}^{M} d_{m}\left(t, C_{i}\right)>\sum_{m=1}^{M} d_{m}\left(t, C_{r}\right)\right)\right) \geq \\
\prod_{i \neq r, i=1}^{k}\left(P\left(d_{m}\left(t, C_{i}\right)>d_{m}\left(t, C_{r}\right)\right)\right) .
\end{gathered}
$$

According to the definitions of $\psi_{m}\left(t, C_{r}\right)$ and $\Psi\left(t, C_{r}\right)$, we have

$$
\psi_{m}\left(t, C_{r}\right)=\prod_{i \neq r, i=1}^{k} P\left(d_{m}\left(t, C_{i}\right)-d_{m}\left(t, C_{r}\right)>0\right),
$$

and

$$
\Psi\left(t, C_{r}\right)=\prod_{i \neq r, i=1}^{k} P\left(\sum_{m=1}^{M} d_{m}\left(t, C_{i}\right)-\sum_{m=1}^{M} d_{m}\left(t, C_{r}\right)>0\right),
$$

by combining Eqs 11, 12 and 13, we have

$$
\Psi\left(t, C_{r}\right) \geq \psi_{m}\left(t, C_{r}\right) \cdot \square
$$

\section{Experimental Results and Discussion}

We compared image classification accuracy of ADM, BDM and bagging. For the bagging approach, BDM classification was done for each sub-set before a plurality vote. Both clinical and non-clinical images were used. We directly used image pixel intensities in the metric learning, so that the results obtained in this way would not depend on any specific image feature. We also performed preliminary experiments on the potential application of ADM in image retrieval. In order to do so, following the notation in $\mathrm{Eq} 3$, we define the aggregated distance between $t$ and $x_{i j}$ as $D\left(t, x_{i j}\right)=\sum_{m=1}^{M} d_{m}\left(t, x_{i j}\right)$ and employ this measure for image retrieval. 
Table 1. Comparison of classification accuracy of clinical images using different methods (ADM, BDM and bagging). The highest accuracy values are shown bolded.

\begin{tabular}{c|c|c|c|c|c}
\hline \multirow{2}{*}{$\begin{array}{c}\text { Number of } \\
\text { classes }(k)\end{array}$} & $\begin{array}{c}\text { Number of training } \\
\text { image per class }(n)\end{array}$ & $\begin{array}{c}\text { Number of } \\
\text { test image }\end{array}$ & \multicolumn{3}{|c}{ Classification accuracy } \\
\hline \hline 6 & 3 & 68 & $\mathbf{0 . 5 0}$ & 0.31 & 0.38 \\
\hline 10 & 3 & 106 & $\mathbf{0 . 3 9}$ & 0.12 & 0.22 \\
\hline 8 & 4 & 80 & $\mathbf{0 . 4 3}$ & 0.27 & 0.38 \\
\hline 14 & 4 & 128 & $\mathbf{0 . 3 9}$ & 0.11 & 0.25 \\
\hline 10 & 5 & 86 & $\mathbf{0 . 4 3}$ & 0.30 & 0.36 \\
\hline 10 & 6 & 76 & $\mathbf{0 . 6 9}$ & 0.66 & 0.68 \\
\hline
\end{tabular}

\subsection{Experiments Using Clinical Images}

The clinical images used are the X-ray images from ImageCLEFmed2009 data set1, which consists of 12677 images that have already been categorized into 193 classes by experts. We randomly selected images of $k$ classes, and for each class, $n$ images were randomly chosen as the training images while the remaining images were used as the test images. The classification of these test images were already known, which could be used as the ground truth to calculate the classification accuracy. We varied the values of $k$ and $n$ to change the training data size.

Experiments on image classification: For each set of $k$ and $n$, image classification experiments were done using ADM, BDM, and bagging, respectively. Table 1 shows the result. For all the different values of $k$ and $n$, our method achieved the highest classification accuracy. Note that although these accuracy values are lower than those reported in [1, a significantly smaller number of training images was employed in this study.

Preliminary experiments on image retrieval: ADM and BDM were also compared in the context of image retrieval. We randomly selected test image as the query image to retrieve images in the training data. Figure 1 shows a few examples of the retrieval when $k=6$ and $n=3$, where only the 3 closest matches found by ADM and BDM are shown. Our method gave a better result as the retrieved images contains fewer irrelevant results.

\subsection{Experiments Using Non-clinical Images}

The non-clinical images used are the toy car images2, which consists of 255 images of 14 classes. Like experiments using clinical images, the values of $k$ and $n$ are varied to change the size of training data.

Experiments on image classification: ADM was compared with BDM and bagging for each set of $k$ and $n$ in the same manner as for the clinical images. All color images were turned into grayscale before the experiments. Table 2

${ }^{1}$ http://ganymed.imib.rwth-aachen.de/irma/datasets_en.php

${ }^{2}$ http://lear.inrialpes.fr/people/nowak/dwl/toycarlear.tar.gz 


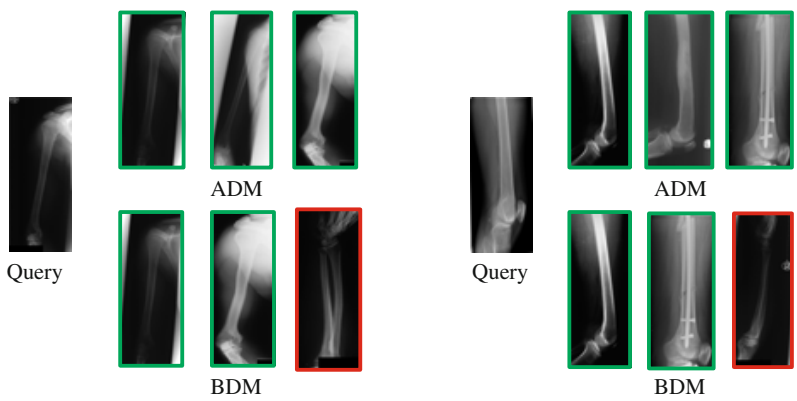

Fig. 1. Examples of clinical image retrieval result. Green and red bounding boxes indicate relevant and irrelevant retrieval result, respectively. Retrieved images are sorted according to their similarity to the query.

Table 2. Classification accuracy of non-clinical images using different methods. The highest accuracy values are shown bolded.

\begin{tabular}{c|c|c|c|c|c}
\hline Number of & Number of training & Number of & \multicolumn{3}{|c}{ Classification accuracy } \\
\cline { 4 - 6 } classes $(k)$ & image per class $(n)$ & test images & ADM & BDM & Bagging \\
\hline \hline 6 & 3 & 94 & $\mathbf{0 . 8 6}$ & 0.78 & 0.79 \\
\hline 10 & 3 & 160 & $\mathbf{0 . 6 4}$ & 0.51 & 0.46 \\
\hline 14 & 4 & 200 & $\mathbf{0 . 6 1}$ & 0.46 & 0.41 \\
\hline 10 & 6 & 130 & $\mathbf{0 . 6 9}$ & 0.66 & 0.67 \\
\hline
\end{tabular}

shows the comparison result. For all the different values of $k$ and $n$, our method returned fewer irrelevant images.

Preliminary experiments on image retrieval: We also compared ADM and BDM in the context of image retrieval. Query image was randomly chosen to retrieve the training images. Figure 2 shows a few examples of the retrieval when $k=6$ and $n=3$, where only the 3 closest matches found by ADM and BDM are shown. Our method again gave a better result.
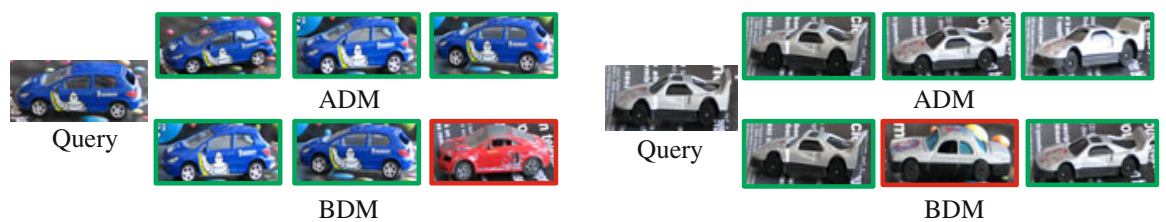

Fig. 2. Two examples of non-clinical image retrieval result. Green and red bounding boxes indicate relevant and irrelevant retrieval result, respectively. Retrieved images are sorted according to their similarity to the query. 


\subsection{Discussion}

We found that our method performs better than bagging when the training data for each class are limited. We suspect the reason is that the plurality vote, which is employed by bagging, sometimes results in two or more classes tied in a vote. In such cases, bagging selects a class randomly. However, when evaluated by the summation of numeric distances, as in our method, it is less likely to result in a tie.

In Section 3.2, at each iteration, we randomly choose $n$ whole classes of images to construct the sub-sets. We would like to point out that theoretically, there should be no restriction on how the sub-sets are constructed. The reason behind our choice is that all the training images of a certain class can be utilized. Since the number of training images for each class is already small, further reducing this number may run the risk of decreasing the statistical representativeness of the data even more [4. Also, in this way, we manage to place a reasonable upper limit on the total number of sub-sets.

\section{Concluding Remarks}

Accurate image classification is important in many medical applications. Supervised distance metric learning has shown great potential in image classification tasks. However, when faced with limited training data, especially when the number of training images for each class is small, the classification accuracy may be severely affected. We presented a novel method called aggregated distance metric learning $(\mathrm{ADM})$ to classify a test image with limited number of training images. Our method combines the best of distance metric learning and bagging. Our method was found to be more accurate compared to both BDM and the bagging approach. We also presented a rigorous theoretical analysis to demonstrate that ADM is better at image classification compared to BDM. Experimental results using both clinical and non-clinical image data showed the efficacy of our method. Future work will involve further testing of our method on additional data sets and performing more extensive quantitative evaluation.

\section{References}

1. Yang, L., Jin, R., Mummert, L., Sukthankar, R., Goode, A., Zheng, B., Hoi, S., Satyanarayanan, M.: A boosting framework for visuality-preserving distance metric learning and its application to medical image retrieval. IEEE PAMI 32, 30-44 (2010)

2. Xing, E., Ng, A., Jordan, M., Russell, S.: Distance metric learning, with application to clustering with side-information. In: NIPS, pp. 521-528 (2002)

3. Hertz, T., Hillel, A., Weinshall, D.: Learning a kernel function for classification with small training samples. In: ICML, pp. 401-408 (2006)

4. Dekel, O., Shamir, O.: Multiclass-multilabel classification with more classes than examples. In: International Conference on Artificial Intelligence and Statistics, pp.137144 (2010)

5. Zhang, T.: Class-size independent generalization analysis of some discriminative multi-category classification. In: NIPS, pp. 1625-1632 (2004)

6. Breiman, L.: Bagging predictors. Machine Learning 24, 123-140 (1996) 